INPLASY

PROTOCOL

To cite: Xu et al. Efficacy and safety of floating needle therapy in the treatment of post-stroke shoulder-hand

Syndrome, a protocol for

Systematic Review and Meta-

Analysis. Inplasy protocol

202210012. doi:

10.37766/inplasy2022.1.0012

Received: 03 January 2022

Published: 03 January 2022

Corresponding author:

Qian Xu

xuqian36@126.com

Author Affiliation:

Jiangsu Province Hospital of

Chinese Medicine, Affiliated

Hospital of Nanjing University

of Chinese Medicine.

Support: Foundation No.

81803859.

Review Stage at time of this submission: The review has not yet started.

Conflicts of interest:

None declared.

\section{Efficacy and safety of floating needle therapy in the treatment of post-stroke shoulder-hand Syndrome, a protocol for Systematic Review and Meta-Analysis}

Xu, Q1; Zhu, J2; Zou, R³.

Review question / Objective: This systematic review aimed to comprehensively evaluate the safety and efficacy of floating needle trerapy for Shoulder-hand syndrome (SHS) in stroke patients.

Condition being studied: Shoulder-hand syndrome (SHS) is a common condition among people who have had a stroke, with its reported prevalence ranging from $12 \%$ to $49 \%$. The main symptoms of SHS include pain, hyperalgesia, joint swelling and limitations in range of motion (ROM). Post-stroke SHS is also named type I complex regional pain syndrome (CRPS) or reflex sympathetic dystrophy. The key to effectively treating SHS is believed to be an expert multidisciplinary team that provides individualized therapy. There is a wide range of treatment options available to help manage post-stroke SHS, including physical therapy, medications, regional anesthesia techniques and neuromodulation. However, there is insufficient evidence to support their efficacy.

INPLASY registration number: This protocol was registered with the International Platform of Registered Systematic Review and Meta-Analysis Protocols (INPLASY) on 03 January 2022 and was last updated on 03 January 2022 (registration number INPLASY202210012).

\section{INTRODUCTION}

Review question / Objective: This systematic review aimed to comprehensively evaluate the safety and efficacy of floating needle trerapy for
Shoulder-hand syndrome (SHS) in stroke patients.

Rationale: Shoulder-hand syndrome (SHS) is a common condition among people who have had a stroke, with its reported prevalence ranging from $12 \%$ to $49 \%$. The 
main symptoms of SHS include pain, hyperalgesia, joint swelling and limitations in range of motion (ROM). Post-stroke SHS is also named type I complex regional pain syndrome (CRPS) or reflex sympathetic dystrophy. The key to effectively treating SHS is believed to be an expert multidisciplinary team that provides individualized therapy. There is a wide range of treatment options available to help manage post-stroke SHS, including physical therapy, medications, regional anesthesia techniques and neuromodulation. However, there is insufficient evidence to support their efficacy. With the popularization and application of floating needle therapy in clinical practice, more and more literatures have been reported on the clinical efficacy of floating needle therapy in the treatment of post-stroke SHS, and a large number of literatures have shown that floating needle therapy has a good effect in the treatment of post-stroke SHS. However, there is still a lack of systematic evaluation on the efficacy and safety of floating needle therapy for post-stroke SHS in clinical practice. Therefore, the effectiveness and safety of floating needle therapy in the treatment of post-stroke SHS will be systematically evaluated and metaanalyzed in this paper.

Condition being studied: Shoulder-hand syndrome (SHS) is a common condition among people who have had a stroke, with its reported prevalence ranging from $12 \%$ to $49 \%$. The main symptoms of SHS include pain, hyperalgesia, joint swelling and limitations in range of motion (ROM). Post-stroke SHS is also named type I complex regional pain syndrome (CRPS) or reflex sympathetic dystrophy. The key to effectively treating SHS is believed to be an expert multidisciplinary team that provides individualized therapy. There is a wide range of treatment options available to help manage post-stroke SHS, including physical therapy, medications, regional anesthesia techniques and neuromodulation. However, there is insufficient evidence to support their efficacy.

\section{METHODS}

Search strategy: We will search articles in three English databases including PubMed, EMBASE and Cochrane Library, and four Chinese databases (CBM, CNKI, CQVIP, and Wanfang). All the publications until January 2022 will be searched without any restriction of countries . Randomized, controlled trials that evaluated the effect of floating needle to rehabilitation for poststroke SHS were identified. Reference list of all selected articles will independently screened to identify additional studies left out in the initial search.

Participant or population: We limited participants to people who were diagnosed with post-stroke SHS. The stroke (ischemic or hemorrhagic) diagnosis needed to be confirmed by computer tomography or magnetic resonance imaging. The SHS or CRPS type I diagnosis was based on clinical symptoms, including pain, motor disturbances and skin changes.

Intervention: The rehabilitation therapy used in RCTs or quasi-RCTs could be a combination of physiotherapy and occupational therapy, such as active range of motion(ROM), passive ROM, mirror visual feedback, Bobath therapy, alternating heat and cold baths, and massage.

Comparator: The comparator was the same as the rehabilitation therapy used in the intervention group, but without floating needle therapy.

Study designs to be included: This systematic review included randomized controlled trials (RCT) or quasi-RCTs that were published in English or Chinese.

Eligibility criteria: This systematic review included randomized controlled trials (RCT) or quasi-RCTs that were published in English or Chinese and evaluated effect of floating needle as an additional therapy for post-stroke SHS. We limited participants to people who were diagnosed with poststroke SHS. We included RCTs or quasiRCTs that evaluated the effects of floating 
needle combined with routine care or rehabilitation as the experimental intervention in this review.

Information sources: Articles will be from three English databases including PubMed, EMBASE and Cochrane Library, and four Chinese databases (CBM, CNKI, CQVIP, and Wanfang).

Main outcome(s): (1) motor function: FuglMeyer Assessment (FMA) upper limb; (2) pain assessment using visual analog scale (VAS) or numerical rating scale (NRS).

Additional outcome(s): (1) Barthel Index (BI) or Modified Barthel Index (MBI), which is assessed for self-care and activities of daily living; (2) ROM; and (3) adverse events.

Data management: Data analyses were performed in a random-effects model using the RevMan software. Dichotomous data was reported as relative risk (RR) with corresponding $95 \%$ confidence intervals (CI). For continuous data, the mean difference (MD) with $95 \% \mathrm{Cl}$ was calculated.

Quality assessment / Risk of bias analysis: Two researchers used the Cochrane risk of bias tool to assess the methodological quality of the included studies. They assessed seven items: sequence generation, allocation concealment, blinding of participants and personnel, blinding of outcome assessors, incomplete outcome data, selective reporting, and other sources of bias. The studies were judged as having a "low," "high," or "unclear" risk of bias. They resolved any difference in their assessment of a study by discussing it with a third researcher.

Strategy of data synthesis: Two researchers independently screened the titles, abstracts and full text of studies to assess eligibility. Any uncertainty was resolved through discussion with a third researcher. They extracted data independently and cross-checked it for accuracy. Extracted data included: author, publication year, diagnostic criteria, duration of disease, sample size, participants' age, details of interventions and all clinical outcomes.

Subgroup analysis: If heterogeneity exists in the metaanalysis, the source of heterogeneity should be sought, such as whether the degree of disease, treatment cycle, treatment time of each floating needle, type of intervention, and so on, is the source of heterogeneity. If so, a subgroup analysis should be conducted for these reasons to see whether heterogeneity still exists after analysis.

Sensitivity analysis: Sensitivity analysis can not only assess the stability and reliability of the combined results, but also assess whether the combined results are significantly changed by the influence of a single study. If sufficient literature is included, we will adopt the method of excluding literature one by one, excluding each included study one by one before effect-size combination, changing the inclusion and exclusion criteria or excluding certain types of literature before effect-size combination.

Language: Articles published in English or Chinese language will be searched.

\section{Country(ies) involved: China.}

Keywords: floating needle therapy, poststroke shoulder-hand Syndrome, protocol, systematic review and meta-analysis.

\section{Contributions of each author:}

Author 1 - Qian Xu - The author drafted the manuscript.

Author 2 - Juan Zhu - The author provided statistical expertise.

Author 3 - Rong Zou - The author contributed to the development of the selection criteria, and the risk of bias assessment strategy. 